九州大学学術情報リポジトリ

Kyushu University Institutional Repository

\title{
NONPARAMETRIC ESTIMATION OF LINEAR MULTIPLIER FOR STOCHASTIC DIFFERENTIAL EQUATIONS DRIVEN BY FRACTIONAL LÉVY PROCESS WITH SMALL NOISE
}

\section{B. L. S. Prakasa Rao}

Advanced Institute of Mathematics, Statistics, and Computer Science, Hyderabad, India

https://doi.org/10.5109/4150376

出版情報: Bulletin of informatics and cybernetics. 52 (3)，pp.1-14，2020. 統計科学研究会 バージョン：

権利関係 : 
NONPARAMETRIC ESTIMATION OF LINEAR MULTIPLIER FOR STOCHASTIC DIFFERENTIAL EQUATIONS DRIVEN BY

FRACTIONAL LÉVY PROCESS WITH SMALL NOISE

by

B.L.S. Prakasa Rao

Reprinted from the Bulletin of Informatics and Cybernetics

Research Association of Statistical Sciences, Vol.52, No. 3

FUKUOKA, JAPAN

2020 


\title{
NONPARAMETRIC ESTIMATION OF LINEAR MULTIPLIER FOR STOCHASTIC DIFFERENTIAL EQUATIONS DRIVEN BY FRACTIONAL LÉVY PROCESS WITH SMALL NOISE
}

\author{
By
}

\section{B.L.S. Prakasa RaO*}

\begin{abstract}
We discuss nonparametric estimation of the linear multiplier in a trend coefficient in models governed by a stochastic differential equation driven by a fractional Lévy process with small noise.
\end{abstract}

Key Words and Phrases: Stochastic differential equation; Linear multiplier; Trend coefficient; Nonparametric estimation; Kernel method; Small noise; fractional Lévy process.

\section{Introduction}

Statistical inference for fractional diffusion processes satisfying stochastic differential equations driven by a fractional Brownian motion ( $\mathrm{fBm}$ ) has been studied earlier and a comprehensive survey of various methods is given in Mishura [9] and Prakasa Rao [12]. There has been a recent interest to study similar problems for stochastic processes driven by $\alpha$-stable noises and by fractional Lévy processes.

Prakasa Rao [11] investigated minimum $L_{1}$-norm estimation for fractional OrnsteinUhlenbeck type process driven by a fractional Brownian motion. Diop and Yode [3] studied minimum distance parameter estimation for Ornstein-Uhlenbeck processes driven by a Lévy process. Parametric estimation for Ornstein-Uhlenbeck process driven by fractional Lévy process is discussed in Shen et al. [16].

In modeling processes with possible long range dependence, it is possible that no special functional form is available for modeling the trend a priori and it is necessary to estimate the trend function based on the observed process over an interval. This problem of estimation is known as nonparametric function estimation in classical statistical inference (cf. Prakasa Rao [10]).

Nonparametric estimation of the trend for stochastic differential equations driven by fractional Brownian motion is investigated in Mishra and Prakasa Rao [8]. Following techniques in Mishra and Prakasa Rao [8], Zhang et al. [17] studied a similar problem when the driving force is a small $\alpha$-stable noise. Nonparametric estimation of trend for stochastic differential equations driven by a fractional Lévy process is investigated in Prakasa Rao [13].

\footnotetext{
* CR RAO Advanced Institute of Mathematics, Statistics, and Computer Science, Hyderabad, India.
} 
Our aim im this paper is to study nonparametric estimation of the linear multiplier in the trend function when the process is governed by a stochastic differential equation driven by a fractional Lévy process following the ideas of density function estimation and regression function estimation in classical statistical inference. Several methods are present for nonparametric function estimation as described in Prakasa Rao [10]. The method of kernels is widely used for the estimation of a density function or a regression function and it is known the properties of such an estimator do not depend on the choice of the kernel in general but on the choice of the bandwidth. Properties of the estimators of a density function and a regression function, using the method of kernels, are described in Prakasa Rao [10]. Our aim is to propose a kernel type estimator for the linear multiplier in the trend function and study its properties. We will show that the kernel type estimator is uniformly consistent over a class of trend functions and obtain the asymptotic distribution of the estimator in the presence of small noise. We will also obtain the optimum rate of convergence of the kernel type estimators for the trend function. Results derived in this paper will be useful when there is no information on the functional form of the trend coefficient and the trend has to be estimated from the observed path of the underlying process.

We define a fractional Lévy process (fLp) in Section 2 and describe its properties. Section 3 contains a preliminary description of processes driven by a fLp with small noise. Estimation of the trend function by the kernel method is suggested and results on its rate of convergence is investigated in Section 4. Proofs of these results are given in Section 5. An alternate estimator of the linear multiplier based on a modified or a second stage sampling process via kernel method is described and its properties are discussed in Section 6.

\section{Fractional Lévy Process}

We will now describe some properties of a fractional Lévy process (fLp) and properties of processes driven by a fractional Lévy process. A fractional Lévy process is a generalization of the integral representation of fractional Brownian motion. For any $t \in R$, define $t_{+}=\max (t, 0)$ and $t_{-}=\max (-t, 0)$.

Definition: (Marquardt [7]) Let $\left\{L_{1}(t), t \geq 0\right\}$ and $\left\{L_{2}(t), t \geq 0\right\}$ be two independent copies of a one-sided zero mean Lévy process. Let $L(t)=L_{1}(t), t \geq 0$ and $L(t)=$ $-L_{2}\left(-t_{-}\right), t<0$. Further suppose that $E\left([L(1)]^{2}\right)<\infty$ and the process $\{L(t), t \in R\}$ has no Brownian component. For $d \in\left(0, \frac{1}{2}\right)$, define the stochastic process

$$
L_{t}^{d}=\frac{1}{\Gamma(d+1)} \int_{-\infty}^{\infty}\left[(t-s)_{+}^{d}-(-s)_{+}^{d}\right] L(d s), t \in R .
$$

This process is called a fractional Lévy process .

Suppose the process $L_{1}$ is a Lévy process with $E\left[L_{1}(1)\right]=0$ without Brownian component. Further suppose that $E\left[L_{1}(1)\right]^{2}<\infty$. This in turn implies that $E\left[L_{1}(t)\right]^{2}<\infty$ since $\operatorname{Var}\left(L_{1}(t)\right)=t \operatorname{Var}\left(L_{1}(1)\right.$ (cf. Sato [15], Example 25.12; Marquardt [7], Equation (2.6)). The following two results are due to Marquardt [7].

Theorem 2.1: Let the function $g \in H$ where $H$ is the completion of $L^{1}(R) \cap L^{2}(R)$ 
with respect to the norm $\|g\|_{H}^{2}=\frac{E\left([L(1)]^{2}\right)}{\int_{R}\left(I_{g}^{d}\right)^{2}(u) d u}$. Then

$$
\int_{R} g(s) d L_{s}^{d}=\int_{R}\left(I_{g}^{d}\right)(u) d L(u)
$$

where the equality holds in the $L^{2}$-sense and $I_{g}^{d}$ denotes the Riemann-Liouville fractional integral defined by

$$
\left(I_{g}^{d}\right)(x)=\frac{1}{\Gamma(d)} \int_{x}^{\infty} g(t)(t-x)^{d-1} d t
$$

Theorem 2.1 gives a representation of the integral with respect to a fractional Lévy process (fLp) as an integral with respect to a transformed function with respect to a Lévy process. The next result gives a formula for the product moment of two integrals with respect to fractional Lévy process.

Theorem 2.2: Let $|f|,|g| \in H$. Then

$$
E\left(\int_{R} f(s) d L_{s}^{d}\right)=0
$$

and

$$
E\left[\int_{R} f(s) d L_{s}^{d} \int_{R} g(s) d L_{s}^{d}\right]=\frac{\Gamma(1-2 d) E\left([L(1)]^{2}\right)}{\Gamma(d) \Gamma(1-d)} \int_{R} \int_{R} f(t) g(s)|t-s|^{2 d-1} d s d t .
$$

Bender et al. [1] presented a maximal inequality for a fractional Lévy process.

Theorem 2.3: Let $\left\{L_{t}^{d}, t \in R\right\}$ be a fractional Lévy process. Then, for every $p \geq 2$ and $\delta>0$ such that $d+\delta<\frac{1}{2}$, there exists a constant $C_{p, \delta, d}$ independent of the Lévy process $L$ such that for every $T \geq 1$,

$$
E\left(\sup _{0 \leq t \leq T}\left|L_{t}^{d}\right|^{p}\right) \leq C_{p, \delta, d} E\left(|L(1)|^{p}\right) T^{p\left(d+\frac{1}{2}+\delta\right)} .
$$

Remarks : It is known that a fractional Lévy process (fLp) is not a semimartingale in general for a broad class of Lévy processes and hence it is not possible to extend the notion of the Ito stochastic integral for stochastic integrals with respect to a fractional Lévy process. However it is possible to extend the notion of a Wiener integral with respect to a fLp when the integrand is a non-random function using the ideas from fractional calculus. The covariance structure of $f L p$ is almost the same as that of a fractional Brownian motion. In fact,

$$
\operatorname{Cov}\left(L_{t}^{d}, L_{s}^{d}\right)=\frac{E\left[L(1)^{2}\right]}{2 \Gamma(2 d+2) \sin \left(\pi\left(d+\frac{1}{2}\right)\right)}\left[|t|^{2 d+1}-|t-s|^{2 d+1}+|s|^{2 d+1}\right] .
$$

Furthermore the increments of a fLp are stationary and exhibit long memory. Its sample paths are Hölder continuous of order $\beta<d$ and the fLp is not self-similar. For details, see Marquardt [7]. For additional properties of fractional Lévy processes, see Bender et al. [2], Fink and Kluppelberg [5], and Engelke [4]. 


\section{Preliminaries}

Let us consider the stochastic differential equation

$$
d X_{t}=\theta(t) X_{t} d t+\epsilon d L_{t}^{d}, X_{0}=x_{0}, 0 \leq t \leq T
$$

where the linear multiplier $\theta($.$) is an unknown function and the constant d$ is known with $0<d<\frac{1}{2}$. We assume that $T \geq 1$ hereafter. Suppose $\left\{x_{t}, 0 \leq t \leq T\right\}$ is the solution of the differential equation

$$
\frac{d x_{t}}{d t}=\theta(t) x_{t}, x_{0}, 0 \leq t \leq T .
$$

We would like to estimate the trend $\theta(t) x_{t}$ based on the observation $\left\{X_{t}, 0 \leq t \leq T\right\}$.

$\left(A_{1}\right)$ We assume that the linear multiplier $\theta($.$) is bounded by a constant L$.

Lemma 3.1: Let $X_{t}$ and $x_{t}$ be the solutions of the equation (3.1) and (3.2) respectively. Let $\delta>0$ such that $d+\delta<\frac{1}{2}$ and $T \geq 1$. Then, with probability one,

$$
(a)\left|X_{t}-x_{t}\right|<e^{L t} \epsilon \sup _{0 \leq s \leq t}\left|L_{s}^{d}\right|
$$

and, for $T \geq 1$, there exists a constant $C_{2, \delta, d}$ such that

$$
\text { (b) } \sup _{0 \leq t \leq T} E\left|X_{t}-x_{t}\right|^{2} \leq C_{2, \delta, d} E\left[(L(1))^{2}\right] e^{2 L T} \epsilon^{2} T^{2\left(d+\frac{1}{2}+\delta\right)} .
$$

Proof of (a) : Let $u_{t}=\left|X_{t}-x_{t}\right|$. Then

$$
\begin{aligned}
u_{t} & \leq \int_{0}^{t}\left|\theta(v)\left(X_{v}-x_{v}\right)\right| d v+\epsilon\left|L_{t}^{d}\right| \\
& \leq L \int_{0}^{t} u_{v} d v+\epsilon \sup _{0 \leq s \leq t}\left|L_{s}^{d}\right| .
\end{aligned}
$$

Applying the Gronwall's lemma, it follows that

$$
u_{t} \leq \epsilon \sup _{0 \leq s \leq t}\left|L_{s}^{d}\right| e^{L t} .
$$

Proof of (b) : Let $T \geq 1$. Applying Theorem 2.3, it follows that

$$
\begin{aligned}
\sup _{0 \leq t \leq T} E\left|X_{t}-x_{t}\right|^{2} & \leq e^{2 L T} \epsilon^{2} E\left[\left(\sup _{0 \leq s \leq T}\left|L_{s}^{d}\right|\right)^{2}\right] \\
& \leq C_{2, \delta, d} E\left[(L(1))^{2}\right] e^{2 L T} \epsilon^{2} T^{2\left(d+\frac{1}{2}+\delta\right)}
\end{aligned}
$$




\section{Main Results}

Let $\Theta_{0}(L)$ denote the class of all functions $\theta($.$) with the same bound L$. Let $\Theta_{k}(L)$ denote the class of all functions $\theta($.$) which are uniformly bounded by the same constant$ $L$ and which are $k$-times differentiable with respect to $t$ satisfying the condition

$$
\left|\theta^{(k)}(x)-\theta^{(k)}(y)\right| \leq L_{1}|x-y|, x, y \in R
$$

for some constant $L_{1}>0$. Here $g^{(k)}(x)$ denotes the $k$-th derivative of $g($.$) at x$ for $k \geq 0$. If $k=0$, we interpret $g^{(0)}$ as $g$.

Let $G(u)$ be a bounded function with compact support $[A, B]$ with $A<0<B$ satisfying the condition

$\left(A_{2}\right) \int_{A}^{B} G(u) d u=1$

It is obvious that the following conditions are satisfied by the function $G($.$) :$

(i) $\int_{-\infty}^{\infty}|G(u)|^{2} d u<\infty$;

(ii) $\int_{-\infty}^{\infty}\left|u^{k+1} G(u)\right|^{2} d u<\infty$.

We define a kernel type estimator $\hat{\theta}_{t}$ of the function $\theta(t)$ by the relation

$$
\widehat{\theta}_{t} X_{t}=\frac{1}{\varphi_{\epsilon}} \int_{0}^{T} G\left(\frac{\tau-t}{\varphi_{\epsilon}}\right) d X_{\tau}
$$

where the normalizing function $\varphi_{\epsilon} \rightarrow 0$ as $\epsilon \rightarrow 0$. Let $E_{\theta}($.$) denote the expectation$ when the function $\theta($.$) is the linear multiplier.$

Theorem 4.1: Suppose that the linear multiplier $\theta(.) \in \Theta_{0}(L)$ and the function $\varphi_{\epsilon} \rightarrow 0$ and $\epsilon^{2} \varphi_{\epsilon}^{2 d-1} \rightarrow 0$ as $\epsilon \rightarrow 0$. Let $T \geq 1$. Suppose the conditions $\left(A_{1}\right)-\left(A_{2}\right)$ hold.Then, for any $0<a \leq b<T$, the estimator $\hat{\theta}_{t}$ is uniformly consistent, that is,

$$
\lim _{\epsilon \rightarrow 0} \sup _{\theta(.) \in \Theta_{0}(L)} \sup _{a \leq t \leq b} E_{\theta}\left(\left|\hat{\theta}_{t} X_{t}-\theta(t) x_{t}\right|^{2}\right)=0 .
$$

In addition to the conditions $\left(A_{1}\right)$ and $\left(A_{2}\right)$, suppose the following condition holds. $\left(A_{3}\right) \int_{-\infty}^{\infty} u^{j} G(u) d u=0$ for $j=1,2, \ldots k$.

Theorem 4.2: Suppose that the function $\theta(.) \in \Theta_{k+1}(L)$ and the conditions $\left(A_{1}\right)-\left(A_{3}\right)$ hold. Further suppose that $\varphi_{\epsilon}=\epsilon^{\frac{2}{2 k-2 d+3}}$. Then,

$$
\limsup _{\epsilon \rightarrow 0} \sup _{\theta(.) \in \Theta_{k+1}(L)} \sup _{a \leq t \leq b} E_{\theta}\left(\left|\hat{\theta}_{t} X_{t}-\theta(t) x_{t}\right|^{2}\right) \epsilon^{-\frac{4(k+1)}{2 k-2 d+3}}<\infty .
$$

Theorem 4.3: Suppose that the function $\theta(.) \in \Theta_{k+1}(L)$ for some $k>1$ and the conditions $\left(A_{1}\right)-\left(A_{3}\right)$ hold. Further suppose that $\varphi_{\epsilon}=\epsilon^{\frac{1}{k+2-\left(d+\frac{1}{2}\right)}}$. Let $J(t)=\theta(t) x_{t}$. 
Then, as $\epsilon \rightarrow 0$, the asymptotic distribution of

$$
\epsilon^{\frac{-(k+1)}{k+2-\left(d+\frac{1}{2}\right)}}\left(\hat{\theta}_{t} X_{t}-J(t)-\frac{J^{(k+1)}(t)}{(k+1) !} \int_{-\infty}^{\infty} G(u) u^{k+1} d u\right)
$$

has mean zero and variance

$$
\sigma^{2}=\int_{-\infty}^{\infty} \int_{-\infty}^{\infty} G(u) G(v)|u-v|^{2 d-1} d u d v
$$

and the asymptotic distribution is that of the family of random variables

$$
\varphi_{\epsilon}^{-\left(d+\frac{1}{2}\right)} \int_{-\infty}^{\infty} G\left(\frac{\tau-t}{\varphi_{\epsilon}}\right) d L_{\tau}^{d}
$$

as $\epsilon \rightarrow 0$.

\section{Proofs of Theorems}

Proof of Theorem 4.1 : From the inequality

$$
(a+b+c)^{2} \leq 3\left(a^{2}+b^{2}+c^{2}\right), a, b, c \in R,
$$

it follows that

$$
\begin{aligned}
E_{\theta}\left[\left|\hat{\theta}(t) x_{t}-\theta(t) x_{t}\right|^{2}\right]= & E_{\theta}\left[\mid \frac{1}{\varphi_{\epsilon}} \int_{0}^{T} G\left(\frac{\tau-t}{\varphi_{\epsilon}}\right)\left(\theta(\tau) X_{\tau}-\theta(\tau) x_{\tau}\right) d \tau\right. \\
& \left.+\frac{1}{\varphi_{\epsilon}} \int_{0}^{T} G\left(\frac{\tau-t}{\varphi_{\epsilon}}\right) \theta(\tau) x_{\tau} d \tau-\theta(t) x_{t}+\left.\frac{\epsilon}{\varphi_{\epsilon}} \int_{0}^{T} G\left(\frac{\tau-t}{\varphi_{\epsilon}}\right) d L_{\tau}^{d}\right|^{2}\right] \\
\leq & 3 E_{\theta}\left[\left|\frac{1}{\varphi_{\epsilon}} \int_{0}^{T} G\left(\frac{\tau-t}{\varphi_{\epsilon}}\right)\left(\theta(\tau) X_{\tau}-\theta(\tau) x_{\tau}\right) d \tau\right|^{2}\right] \\
& +3 E_{\theta}\left[\left|\frac{1}{\varphi_{\epsilon}} \int_{0}^{T} G\left(\frac{\tau-t}{\varphi_{\epsilon}}\right) \theta(\tau) x_{\tau} d \tau-\theta(t) x_{t}\right|^{2}\right] \\
& +3 \frac{\epsilon^{2}}{\varphi_{\epsilon}^{2}} E_{\theta}\left[\left|\int_{0}^{T} G\left(\frac{\tau-t}{\varphi_{\epsilon}}\right) d L_{\tau}^{d}\right|^{2}\right] \\
= & I_{1}+I_{2}+I_{3} \text { (say). }
\end{aligned}
$$

By the boundedness condition on the function $\theta($.$) , the inequality (3.3) in Lemma 3.1$ and the condition $\left(A_{2}\right)$, and applying the Hölder inequality, it follows that

$$
\begin{aligned}
I_{1} & =3 E_{\theta}\left|\frac{1}{\varphi_{\epsilon}} \int_{0}^{T} G\left(\frac{\tau-t}{\varphi_{\epsilon}}\right)\left(\theta(\tau) X_{\tau}-\theta(\tau) x_{\tau}\right) d \tau\right|^{2} \\
& =3 E_{\theta}\left|\int_{-\infty}^{\infty} G(u)\left(\theta\left(t+\varphi_{\epsilon} u\right) X_{t+\varphi_{\epsilon} u}-\theta\left(t+\varphi_{\epsilon} u\right) x_{t+\varphi_{\epsilon} u}\right) d u\right|^{2}
\end{aligned}
$$




$$
\begin{aligned}
& \leq 3(B-A) \int_{-\infty}^{\infty}|G(u)|^{2} L^{2} E\left|X_{t+\varphi_{\epsilon} u}-x_{t+\varphi_{\epsilon} u}\right|^{2} d u \text { (by using the condition }\left(A_{1}\right) \text { ) } \\
& \leq 3(B-A) \int_{-\infty}^{\infty}|G(u)|^{2} L^{2} \sup _{0 \leq t+\varphi_{\epsilon} u \leq T} E_{\theta}\left|X_{t+\varphi_{\epsilon} u}-x_{t+\varphi_{\epsilon} u}\right|^{2} d u \\
& \leq 3(B-A) L^{2} C_{2, \delta, d} E\left[(L(1))^{2}\right] e^{2 L T} \epsilon^{2} T^{2\left(d+\frac{1}{2}+\delta\right)} \int_{-\infty}^{\infty}|G(u)|^{2} d u \text { (by using (3.4)) }
\end{aligned}
$$

which tends to zero as $\epsilon \rightarrow 0$. For the term $I_{2}$, by the boundedness condition on the function $\theta($.$) , the condition \left(A_{2}\right)$ and the Hölder inequality, it follows that

$$
\begin{aligned}
I_{2} & =3 E_{\theta}\left|\frac{1}{\varphi_{\epsilon}} \int_{0}^{T} G\left(\frac{\tau-t}{\varphi_{\epsilon}}\right) \theta(\tau) x_{\tau} d \tau-\theta(t) x_{t}\right|^{2} \\
& =3\left|\int_{-\infty}^{\infty} G(u)\left(\theta\left(t+\varphi_{\epsilon} u\right) x_{t+\varphi_{\epsilon} u}-\theta(t) x_{t}\right) d u\right|^{2} \\
& \leq 3(B-A) L^{2} \varphi_{\epsilon}^{2} \int_{-\infty}^{\infty}|u G(u)|^{2} d u\left(\text { by }\left(A_{2}\right)\right) .
\end{aligned}
$$

The last term tends to zero as $\epsilon \rightarrow 0$. We will now get an upper bound on the term $I_{3}$. Note that

$$
\begin{aligned}
I_{3} & =3 \frac{\epsilon^{2}}{\varphi_{\epsilon}^{2}} E_{\theta}\left|\int_{0}^{T} G\left(\frac{\tau-t}{\varphi_{\epsilon}}\right) d L_{\tau}^{d}\right|^{2} \\
& =3 \frac{\epsilon^{2}}{\varphi_{\epsilon}^{2}} \frac{\Gamma(1-2 d) E\left[L(1)^{2}\right]}{\Gamma(d) \Gamma(1-d)} \int_{0}^{T} \int_{0}^{T} G\left(\frac{\tau-t}{\varphi_{\epsilon}}\right) G\left(\frac{\tau-s}{\varphi_{\epsilon}}\right)|t-s|^{2 d-1} d s d t \\
& \leq C_{1} \frac{\epsilon^{2}}{\varphi_{\epsilon}^{2}} \varphi_{\epsilon}^{2 d+1} \int_{R} \int_{R} G(u) G(v)|u-v|^{2 d-1} d u d v
\end{aligned}
$$

for some positive constant $C_{1}$. Theorem 4.1 is now proved by using the equations (5.1) to (5.4).

Proof of Theorem 4.2 : Let $J(t)=\theta(t) x_{t}$. By the Taylor's formula, for any $x \in R$,

$$
J(y)=J(x)+\sum_{j=1}^{k} J^{(j)}(x) \frac{(y-x)^{j}}{j !}+\left[J^{(k)}(z)-J^{(k)}(x)\right] \frac{(y-x)^{k}}{k !}
$$

for some $z$ such that $|z-x| \leq|y-x|$. Using this expansion, the equation (3.2) and the condition $\left(A_{3}\right)$ in the expression for $I_{2}$ defined in the proof of Theorem 4.1, it follows that 


$$
\begin{aligned}
I_{2}= & 3\left[\int_{-\infty}^{\infty} G(u)\left(J\left(t+\varphi_{\epsilon} u\right)-J(t)\right) d u\right]^{2} \\
= & 3\left[\sum_{j=1}^{k} J^{(j)}(t)\left(\int_{-\infty}^{\infty} G(u) u^{j} d u\right) \varphi_{\epsilon}^{j}(j !)^{-1}\right. \\
& +\left(\int_{-\infty}^{\infty} G(u) u^{k}\left(J^{(k)}\left(z_{u}\right)-J^{(k)}\left(x_{t}\right)\right) d u \varphi_{\epsilon}^{k}(k !)^{-1}\right]^{2}
\end{aligned}
$$

for some $z_{u}$ such that $\left|x_{t}-z_{u}\right| \leq\left|x_{t+\varphi_{\epsilon} u}-x_{t}\right| \leq C\left|\varphi_{\epsilon} u\right|$. Hence

$$
\begin{aligned}
I_{2} & \leq 3 L^{2}\left[\int_{-\infty}^{\infty}\left|G(u) u^{k+1}\right| \varphi_{\epsilon}^{k+1}(k !)^{-1} d u\right]^{2} \\
& \leq 3 L^{2}(B-A)(k !)^{-2} \varphi_{\epsilon}^{2(k+1)} \int_{-\infty}^{\infty} G^{2}(u) u^{2(k+1)} d u \\
& \leq C_{2} \varphi_{\epsilon}^{2(k+1)}
\end{aligned}
$$

for some positive constant $C_{2}$. Combining the equations (5.2)- (5.5), we get that there exists a positive constant $C_{3}$ such that

$$
\sup _{a \leq t \leq b} E_{\theta}\left|\hat{\theta}_{t} X_{t}-\theta(t) x_{t}\right|^{2} \leq C_{3}\left(\epsilon^{2}+\varphi_{\epsilon}^{2(k+1)}+\epsilon^{2} \varphi_{\epsilon}^{2 d-1}\right)
$$

Choosing $\varphi_{\epsilon}=\epsilon^{\frac{2}{2 k-2 d+3}}$, we get that

$$
\limsup _{\epsilon \rightarrow 0} \sup _{\theta(.) \in \Theta_{k+1}(L)} \sup _{a \leq t \leq b} E_{\theta}\left|\theta(t) X_{t}-\theta(t) x_{t}\right|^{2} \epsilon^{-\frac{4(k+1)}{2 k-2 d+3}}<\infty .
$$

This completes the proof of Theorem 4.2.

Proof of Theorem 4.3: Let $\alpha=\frac{2 k-2}{2 k-2 d+1}$. Observe that $0<\alpha<1$. From (3.1), we obtain that

$$
\begin{aligned}
\epsilon^{-\alpha}\left(\hat{\theta}(t) X_{t}-\theta(t) x_{t}\right) \\
=\quad \epsilon^{-\alpha}\left[\frac{1}{\varphi_{\epsilon}} \int_{0}^{T} G\left(\frac{\tau-t}{\varphi_{\epsilon}}\right)\left(\theta(\tau) X_{\tau}-\theta(\tau) x_{\tau}\right) d \tau\right. \\
\left.\quad+\frac{1}{\varphi_{\epsilon}} \int_{0}^{T} G\left(\frac{\tau-t}{\varphi_{\epsilon}}\right) \theta(\tau) x_{\tau} d \tau-\theta(t) x_{t}+\frac{\epsilon}{\varphi_{\epsilon}} \int_{0}^{T} G\left(\frac{\tau-t}{\varphi_{\epsilon}}\right) d L_{\tau}^{d}\right] \\
=\quad \epsilon^{-\alpha}\left[\int_{-\infty}^{\infty} G(u)\left(\theta\left(t+\varphi_{\epsilon} u\right) X_{t+\varphi_{\epsilon} u}-\theta\left(t+\varphi_{\epsilon} u\right) x_{t+\varphi_{\epsilon} u}\right) d u\right. \\
\quad+\int_{-\infty}^{\infty} G(u)\left(\theta\left(t+\varphi_{\epsilon} u\right) x_{t+\varphi_{\epsilon} u}-\theta(t) x_{t}\right) d u \\
\left.\quad+\frac{\epsilon}{\varphi_{\epsilon}} \int_{0}^{T} G\left(\frac{\tau-t}{\varphi_{\epsilon}}\right) d L_{\tau}^{d}\right] . \\
=R_{1}+R_{2}+R_{3} \text { (say) }
\end{aligned}
$$


By the boundedness condition on the function $\theta($.$) and part (a) of Lemma 3.1, it follows$ that

$$
\begin{aligned}
R_{1} & \leq \epsilon^{-\alpha}\left|\int_{-\infty}^{\infty} G(u)\left(\theta\left(t+\varphi_{\epsilon} u\right) X_{t+\varphi_{\epsilon} u}-\theta\left(t+\varphi_{\epsilon} u\right) x_{t+\varphi_{\epsilon} u}\right) d u\right| \\
& \leq \epsilon^{-\alpha} \epsilon L \int_{-\infty}^{\infty}|G(u)|\left(X_{t+\varphi_{\epsilon} u}-x_{t+\varphi_{\epsilon} u}\right) \mid d u \\
& \leq L e^{L T} \epsilon^{1-\alpha} \int_{-\infty}^{\infty}|G(u)| \sup _{0 \leq t+\varphi_{\epsilon} u \leq T}\left|L_{t+\varphi_{\epsilon} u}^{d}\right| d u
\end{aligned}
$$

Applying the Markov's inequality and Theorem 2.3, for any $\eta>0$,

$$
\begin{aligned}
P\left(\left|R_{1}\right|>\eta\right) & \leq \epsilon^{1-\alpha} \eta^{-1} L e^{L T} \int_{-\infty}^{\infty}|G(u)| E_{\theta}\left(\sup _{0 \leq t+\varphi_{\epsilon} u \leq T}\left|L_{t+\varphi_{\epsilon} u}^{d}\right|\right) d u \\
& \leq \epsilon^{1-\alpha} \eta^{-1} L e^{L T} \int_{-\infty}^{\infty}|G(u)| \mid E_{\theta}\left[\left.\left(\sup _{0 \leq t+\varphi_{\epsilon} u \leq T}\left(L_{t+\varphi_{\epsilon} u}^{d}\right)^{2}\right]\right|^{1 / 2} d u\right. \\
& \leq \epsilon^{1-\alpha} \eta^{-1} L e^{L T} C_{2, \delta, d}^{1 / 2}\left[E_{\theta}\left(|L(1)|^{2}\right)\right]^{1 / 2} T^{\left(d+\frac{1}{2}+\delta\right)} \int_{-\infty}^{\infty}|G(u)| d u .
\end{aligned}
$$

and the last term tends to zero as $\epsilon \rightarrow 0$. Let $J_{t}=\theta(t) x_{t}$. By the Taylor's formula, for any $t \in[0, T]$,

$$
J_{t}=J_{t_{0}}+\sum_{j=1}^{k+1} J_{t_{0}}^{(j)} \frac{\left(t-t_{0}\right)^{j}}{j !}+\left[J_{t_{0}+\gamma\left(t-t_{0}\right)}^{(k+1)}-J_{t_{0}}^{(k+1)}\right] \frac{\left(t-t_{0}\right)^{k+1}}{(k+1) !}
$$

where $0<\gamma<1$ and $t_{0} \in(0, T)$. Applying the Condition $\left(A_{3}\right)$ and the Taylor's expansion, it follows that

$$
\begin{aligned}
R_{2}=\epsilon^{-\alpha} & {\left[\sum_{j=1}^{k+1} J_{t}^{(j)}\left(\int_{-\infty}^{\infty} G(u) u^{j} d u\right) \varphi_{\epsilon}^{j}(j !)^{-1}\right.} \\
& \left.\quad+\frac{\varphi_{\epsilon}^{k+1}}{(k+1) !} \int_{-\infty}^{\infty} G(u) u^{k+1}\left(J_{t+\gamma \varphi_{\epsilon} u}^{(k+1)}-J_{t}^{(k+1)}\right) d u\right] \\
=\epsilon^{-\alpha} & \frac{J_{t}^{(k+1)}}{(k+1) !} \int_{-\infty}^{\infty} G(u) u^{k+1} d u \\
& +\varphi_{\epsilon}^{k+1} \epsilon^{-\alpha} \frac{1}{(k+1) !} \int_{-\infty}^{\infty} G(u) u^{k+1}\left(J_{t+\gamma \varphi_{\epsilon} u}^{(k+1)}-J_{t}^{(k+1)}\right) d u .
\end{aligned}
$$

Observing that $\theta(t) \in \Theta_{k+1}(L)$, we obtain that

$$
\begin{aligned}
& \frac{1}{(k+1) !} \int_{-\infty}^{\infty} G(u) u^{k+1}\left(J_{t+\gamma \varphi_{\epsilon} u}^{(k+1)}-J_{t}^{(k+1)}\right) d u \\
& \leq \frac{1}{(k+1) !} \int_{-\infty}^{\infty}\left|G(u) u^{k+1}\left(J_{t+\gamma \varphi_{\epsilon} u}^{(k+1)}-J_{t}^{(k+1)}\right)\right| d u \\
& \quad \leq \frac{L \varphi_{\epsilon}}{(k+1) !} \int_{-\infty}^{\infty}\left|G(u) u^{k+2}\right| d u .
\end{aligned}
$$


Combining the equations given above, it follows that

$$
\begin{aligned}
& \epsilon^{\frac{-(k+1)}{k+2-\left(d+\frac{1}{2}\right)}}\left(\hat{\theta}_{t} X_{t}-J(t)-\frac{J_{t}^{(k+1)}}{(k+1) !} \int_{-\infty}^{\infty} G(u) u^{k+1} d u\right) \\
& =O_{p}\left(\epsilon^{1-\alpha}\right)+O_{p}\left(\epsilon^{-\alpha} \varphi_{\epsilon}^{k+2}\right)+\epsilon^{1-\alpha} \varphi_{\epsilon}^{-1} \int_{0}^{T} G\left(\frac{\tau-t}{\varphi_{\epsilon}}\right) d L_{t}^{d} .
\end{aligned}
$$

From the choice of $\varphi_{\epsilon}$ and $\alpha$, it follows that

$$
\epsilon^{1-\alpha} \varphi_{\epsilon}^{-1}=\varphi_{\epsilon}^{-\left(d+\frac{1}{2}\right)}
$$

and, by Theorem 2.2 ,

$$
\begin{aligned}
\operatorname{Var}\left[\varphi_{\epsilon}^{-\left(d+\frac{1}{2}\right)} \int_{0}^{T} G\left(\frac{\tau-t}{\varphi_{\epsilon}}\right) d L_{\tau}^{d}\right] \\
=\varphi_{\epsilon}^{-(2 d+1)} \int_{0}^{T} \int_{0}^{T} G\left(\frac{\tau-s}{\varphi_{\epsilon}}\right) G\left(\frac{\tau-t}{\varphi_{\epsilon}}\right)|t-s|^{2 d-1} d s d t \\
=\int_{R} \int_{R} G(u) G(v)|u-v|^{2 d-1} d u d v
\end{aligned}
$$

Applying the Slutsky's theorem and the equations derived above, it can be checked that the random variable

$$
\epsilon^{\frac{-(k+1)}{k+2-\left(d+\frac{1}{2}\right)}}\left(\hat{\theta}_{t} X_{t}-J_{t}-\frac{J_{t}^{(k+1)}}{(k+1) !} \int_{-\infty}^{\infty} G(u) u^{k+1} d u\right)
$$

has a limiting distribution as $\epsilon \rightarrow 0$ as that of the family of random variables

$$
\varphi_{\epsilon}^{-\left(d+\frac{1}{2}\right)} \int_{-\infty}^{\infty} G\left(\frac{\tau-t}{\varphi_{\epsilon}}\right) d L_{\tau}^{d}
$$

as $\epsilon \rightarrow 0$. This completes the proof of Theorem 4.3 .

Remarks: Let $Y=\int_{R}\left(I_{G}^{d}\right)(u) d L(u)$. Following the results in Rajput and Rosinskii [14] and Marquardt [7], it follows that the distribution of $Y$ is infinitely divisible with characteristic function

$$
E\left[e^{i u Y}\right]=\exp \left[\int_{R} \int_{R} e^{i u\left(I_{G}^{d}\right)(s) x}-1-i u\left(I_{G}^{d}\right)(s) \nu(d x) d s\right]
$$

where $\nu($.$) is the Lévy measure corresponding to the process L$. Furthermore $E(Y)=0$ and $E\left(Y^{2}\right)=\left.E\left[L(1)^{2}\right]\left|\int_{R}\right|\left(I_{G}^{d}\right)(s)\right|^{2} d s$.

\section{Alternate Estimator for the Multiplier $\theta($.}

Let $\Theta_{\rho}\left(L_{\gamma}\right)$ be a class of functions $\theta(t)$ uniformly bounded by a known constant $L$ and $k$-times continuously differentiable for some integer $k \geq 1$ with the $k$-th derivative satisfying the Hölder condition of the order $\gamma \in(0,1)$ :

$$
\left|\theta^{(k)}(t)-\theta^{(k)}(s)\right| \leq L_{\gamma}|t-s|^{\gamma}, \rho=k+\gamma
$$


Suppose the process $\left\{X_{t}, 0 \leq t \leq T\right\}$ satisfies the stochastic differential equation given by the equation (3.1) where the linear multiplier is an unknown function in the class $\Theta_{\rho}\left(L_{\gamma}\right)$ and further suppose that $x_{0}>0$ and is known. From the Lemma 3.1, it follows that

$$
\left|X_{t}-x_{t}\right| \leq \epsilon e^{L t} \sup _{0 \leq s \leq T}\left|L_{s}^{d}\right|
$$

Let

$$
A_{t}=\left\{\omega: \inf _{0 \leq s \leq t} X_{s}(\omega) \geq \frac{1}{2} x_{0} e^{-L t}\right\}
$$

and let $A=A_{T}$. Following the technique suggested in Kutoyants [6], p. 156, we define another process $Y$ with the differential

$$
d Y_{t}=\theta(t) I\left(A_{t}\right) d t+\epsilon 2 x_{0}^{-1} e^{L T} I\left(A_{t}\right) d L_{t}^{d}, 0 \leq t \leq T
$$

We will now construct an alternate estimator of the linear multiplier $\theta($.$) based on the$ process $Y$ over the interval $[0, T]$. Define the estimator

$$
\tilde{\theta}(t)=I(A) \frac{1}{\varphi_{\epsilon}} \int_{0}^{T} G\left(\frac{t-s}{\varphi_{\epsilon}}\right) d Y_{s}
$$

where the kernel function $G($.$) satisfies the conditions \left(A_{1}\right)-\left(A_{3}\right)$. Observe that

$$
\begin{aligned}
E|\tilde{\theta}(t)-\theta(t)|^{2}= & E_{\theta} \mid I(A) \frac{1}{\varphi_{\epsilon}} \int_{0}^{T} G\left(\frac{t-s}{\varphi_{\epsilon}}\right)(\theta(s)-\theta(t)) d s \\
& +I\left(A^{c}\right) \theta(t)+\left.I(A) \frac{\epsilon}{\varphi_{\epsilon}} \int_{0}^{T} G\left(\frac{t-s}{\varphi_{\epsilon}}\right) 2 x_{0}^{-1} e^{L T} d L_{s}^{d}\right|^{2} \\
\leq & 3 E_{\theta}\left|I(A) \int_{R} G(u)\left[\theta\left(t+u \varphi_{\epsilon}\right)-\theta(t)\right] d u\right|^{2}+3|\theta(t)|^{2}\left[P\left(A^{c}\right)\right]^{2} \\
& +3 \frac{\epsilon^{2}}{\varphi_{\epsilon}^{2}}\left|E\left[I(A) \int_{0}^{T} G\left(\frac{t-s}{\varphi_{\epsilon}}\right) 2 x_{0}^{-1} e^{L T} d L_{s}^{d}\right]\right|^{2} \\
= & D_{1}+D_{2}+D_{3} . \text { (say). }
\end{aligned}
$$

Applying the Taylor's theorem and using the fact that the function $\theta(t) \in \Theta_{\rho}\left(L_{\gamma}\right)$, it follows that

$$
D_{1} \leq C_{1} \frac{1}{(k+1) !} \varphi_{\epsilon}^{2 \rho} \int_{R}\left|G^{2}(u) u^{2 \rho}\right| d u
$$

Note that, by Lemma 3.1,

$$
\begin{aligned}
P\left(A^{c}\right) & =P\left(\inf _{0 \leq t \leq T} X_{t}<\frac{1}{2} x_{0} e^{-L T}\right) \\
& \leq P\left(\inf _{0 \leq t \leq T}\left|X_{t}-x_{t}\right|+\inf _{0 \leq t \leq T} x_{t}<\frac{1}{2} x_{0} e^{-L T}\right) \\
& \leq P\left(\inf _{0 \leq t \leq T}\left|X_{t}-x_{t}\right|<-\frac{1}{2} x_{0} e^{-L T}\right)
\end{aligned}
$$




$$
\begin{aligned}
& \leq P\left(\sup _{0 \leq t \leq T}\left|X_{t}-x_{t}\right|>\frac{1}{2} x_{0} e^{-L T}\right) \\
& \leq P\left(\epsilon e^{L T} \sup _{0 \leq t \leq T}\left|L_{t}^{d}\right|>\frac{1}{2} x_{0} e^{-L T}\right) \\
& =P\left(\sup _{0 \leq t \leq T}\left|L_{t}^{d}\right|>\frac{x_{0}}{2 \epsilon} e^{-2 L T}\right) \\
& \leq\left(\frac{x_{0}}{2 \epsilon} e^{-2 L T}\right)^{-2} E\left[\sup _{0 \leq t \leq T}\left|L_{t}^{d}\right|^{2}\right] \\
& \leq\left(\frac{x_{0}}{2 \epsilon} e^{-2 L T}\right)^{-2} C_{2, \delta, d} E\left(\mid L(1)^{2}\right) T^{2\left(d+\frac{1}{2}+\delta\right)}
\end{aligned}
$$

by Theorem 2.3 for some positive constant $C_{2, \delta, d}$. The upper bound obtained above and the fact that $|\theta(s)| \leq L, 0 \leq s \leq T$ leads an upper bound for the term $D_{2}$. We have used the inequality

$$
x_{t}=x_{0} \exp \left(\int_{0}^{t} \theta(s) d s\right) \geq x_{0} e^{-L t}
$$

in the computations given above. Applying Theorem 2.1, it follows that

$$
\begin{aligned}
E & {\left[\left|I(A) \int_{0}^{T} G\left(\frac{t-s}{\varphi_{\epsilon}}\right) 2 x_{0}^{-1} e^{L T} d L_{s}^{d}\right|^{2}\right] } \\
& \leq C E\left[\left|\int_{0}^{T} G\left(\frac{t-s}{\varphi_{\epsilon}}\right) d L_{s}^{d}\right|^{2}\right] \\
& =C \operatorname{Var}\left[\int_{0}^{T} G\left(\frac{t-s}{\varphi_{\epsilon}}\right) d L_{s}^{d}\right] \\
& =C \varphi_{\epsilon}^{2 d+1} \int_{R} \int_{R} G(u) G(v)|u-v|^{2 d-1} d u d v
\end{aligned}
$$

for some positive constant $C$ which leads to an upper bound on the term $D_{3}$. Combining the above estimates, it follows that

$$
E|\tilde{\theta}(t)-\theta(t)|^{2} \leq C_{1} \varphi_{\epsilon}^{2 \rho}+C_{2} \epsilon^{4}+C_{3} \epsilon^{2} \varphi_{\epsilon}^{2 d-1}
$$

for some positive constants $C_{i}, i=1,2,3$. Choosing $\varphi_{\epsilon}=\epsilon^{\frac{2}{2 \rho-2 d+1}}$, we obtain that

$$
E|\tilde{\theta}(t)-\theta(t)|^{2} \leq C_{4} \epsilon^{\frac{4 \rho}{2 \rho-2 d+1}}+C_{5} \epsilon^{4}
$$

for some positive constants $C_{4}$ and $C_{5}$. It easy to see that $0<\frac{4 \rho}{2 \rho-2 d+1}<4$ for $\rho>2 d-1$ since $0<d<\frac{1}{2}$. Hence we obtain the following result implying the uniform consistency of the estimator $\tilde{\theta}(t)$ as an estimator of $\theta(t)$ as $\epsilon \rightarrow 0$.

Theorem 6.1: Let $\theta \in \Theta_{\rho}(L)$ where $\rho>2$. Let $\varphi_{\epsilon}=\epsilon^{2 /(2 \rho-2 d+1)}$. Suppose the conditions $\left(A_{1}\right)-\left(A_{3}\right)$ hold. Then, for any interval $[a, b] \subset[0, T]$,

$$
\limsup _{\epsilon \rightarrow 0} \sup _{\theta(.) \in \Theta_{\rho}(L)} \sup _{a \leq t \leq b} E|\tilde{\theta}(t)-\theta(t)|^{2} \epsilon^{-\frac{4 \rho}{2 \rho-2 d+1}}<\infty .
$$


Acknowledgment : The author thanks the referee for his pertinent comments. This work was done with support under the scheme "INSA Senior Scientist" of the Indian National Science Academy at the CR Rao Advanced Institute for Mathematics, Statistics and Computer Science, Hyderabad 500046, India.

\section{References}

[1] Bender, C., Knobloch, R., and Oberacker, P. Maximal inequalities for fractional Lévy and related processes, Stoch. Anal. Appl., 2015, 33: 701-714.

[2] Bender, C., Lindner, A. and Schicks, M. Finite variation of fractional Lévy processes, J. Theor. Probab., 2012, 25: 594-612.

[3] Diop, A. and Yode, A.F. Minimum distance estimation for Ornstein-Uhlenbeck processes driven by Lévy process, Statist. Probab. Lett., 2010, 80: 122-127.

[4] Engelke, S. A unifying approach to fractional Lévy processes, Stoch. Dyn., 2013, 13: 1250017 .

[5] Fink, H. and Kluppelberg, C. Fractional Lévy driven Ornstein-Uhlenbeck processes and stochastic differential equations, Bernoulli, 2011, 17: 484-506.'

[6] Kutoyants, Yu. A. Identification of Dynamical Systems with Small Noise, Dordrecht: Kluwer, 1994.

[7] Marquardt, T. (2006) Fractional Lévy processes with an application to long memory moving average processes, Bernoulli, 2006, 12: 1109-1126.

[8] Mishra, M.N. and Prakasa Rao, B.L.S. Nonparametric estimation of trend for stochastic differential equations driven by fractional Brownian motion, Stat. Infer. Stoch. Proc., 2011, 14: 101-109.

[9] Mishura, Y. Stochastic Calculus for Fractional Brownian Motion and Related Processes, Berlin: Springer, 2008.

[10] Prakasa Rao, B.L.S. Nonparametric Functional Estimation, New York : Academic Press, 1983.

[11] Prakasa Rao, B.L.S. Minimum $L_{1}$-norm estimation for fractional Ornstien-Uhlenbeck type process, Theory Probab. Math. Statist, 2005, 71: 181-189.

[12] Prakasa Rao, B.L.S. Statistical Inference for Fractional Diffusion Processes, London: Wiley, 2010.

[13] Prakasa Rao, B.L.S. Nonparametric estimation of trend for stochastic differential equations driven by fractional Lévy process, Journal of Statistical Theory and Practice, 2020 (to appear).

[14] Rajput, B. and Rosinski, J. Spectral representations of infinitely divisible processes., Probab. Theory and Related Fields, 1989, 82: 451-487.

[15] Sato, K. Lévy Processes and Infinitely Divisible Distributions, Cambridge: Cambrdige University Press, 1999. 
[16] Shen, G., Li, Y., and Gao, Z. Parameter estimation for Ornstein-Uhlenbeck processes driven by fractional Lévy process, Journal of Inequalities and Applications, 2018:356, https://doi.org/10.1186/s13660-018-1951-0.

[17] Zhang, X., Haoran, Y., and Shu, H. Nonparametric estimation of the trend for stochastic differential equations driven by small $\alpha$-stable noises, Statist. Probab. Lett., 2019, 151: 8-16.

Received November 9, 2020

Revised December 21, 2020 